\title{
Using Augmented Reality to Enhance Medical Education in Heart Diseases: Action Design Research
}

\author{
Dimah Alahmadi, Hind Bitar, Hana ALsaadi, Lamees Boker, Linah Alghamdi \\ King Abdulaziz University, Edarah St, Jeddah, Saudi Arabia
}

\begin{abstract}
Augmented reality (AR) is one of the leading communication technologies being investigated and applied in several contexts. Medical training and health education are two of the fields pioneering the innovation of $A R$ to improve the quality of medical outcomes. In this action design research (ADR), we developed an android mobile application called "CardioSim" for medical educational simulation that helps students enhance and improve their understanding and learning process. Functions are implemented to explain specific scenarios of heart failure disease and the symptoms associated with this disease, in addition to the sounds of different heartbeats and models of heart anatomy. CardioSim's usability has been evaluated by 10 experts from the simulation centre using the System Usability Scale (SUS) and the results were acceptable.
\end{abstract}

Keywords - Augmented reality, medical simulation, heart diseases, heart anatomy, medical education, Action design research

\section{Introduction}

AR is a technology that enhances the real world with content generated by a computer.

DOI: $10.18421 /$ TEM103-18

https://doi.org/10.18421/TEM103-18

Corresponding author: Dimah Alahmadi, King Abdulaziz University, Edarah St, Jeddah, Saudi Arabia.

Email: dalahmadi@kau.edu.sa

Received: 13 November 2020.

Revised: 06 July 2021.

Accepted: 12 July 2021.

Published: 27 August 2021.

(c) BY-NC-ND (C) 2021 Dimah Alahmadi et al; published by UIKTEN. This work is licensed under the Creative Commons Attribution-NonCommercial-NoDerivs 4.0 License.

The article is published with Open Access at www.temjournal.com
This content can include images, audio, video, textual information, and three-dimensional (3D) objects. These augmentations can enhance a person's knowledge and understanding of their surroundings [1]. Today, the use of this technology has increased in many fields, including education, entertainment, ecommerce, and marketing.

The usage of augmented reality technology has continuously increased in educational environments. There are many implementations of the AR. For example, the AR is often used in discovery-based learning, such as in museums and astronomical education. Also, the content of books can be supplemented with three-dimensional presentation and interactive learning experiences. Additionally, the AR can be applied in skills training environments, such as in airplane maintenance training. Students can leverage many benefits when learning in AR environments, such as motivation, increased satisfaction, and concentration [2].

According to the World Health Organization (2020), the number one cause of death around the world, at an estimated 17.9 million deaths every year, is cardiovascular diseases (CVDs) [3]. As such, the heart's structural characteristics and functions should be well studied by the future physicians of the world. One way to facilitate understanding is the use of visual presentation, since this organ is difficult to demonstrate [4]. This paper presents the development of an android mobile application called "CardioSim" that helps learners gain a better understanding of the heart and some of its diseases based on identified learning outcomes. The 3D heart object makes it easy to distinguish between different heart failure cases comfortably and interactively. Furthermore, CardioSim allows the learner to assess the targeted learning objective outcomes. Simply, this app helps the users by showing the anatomy of blood flow to and from the heart using AR technology. In addition, it tests students on whether they can identify heart failure based on different shapes and heartbeat sounds in a set of scenarios using AR technology. CardioSim displays three heart cases: the normal heart, mitral stenosis, and mitral incompetence. The 
app also provides self-assessment for learners to assess their achievement according to the learning objective outcomes. This app enables students to gain more knowledge and understanding regarding two heart diseases. The usability of the CardioSim app in this ADR was tested and evaluated using code testing and the System Usability Scale (SUS).

\section{Literature Review}

\section{Heart Anatomy and AR}

One of the most challenging topics to teach in medical and science schools is heart anatomy due to its complex, 3D nature. Students face some challenges in understanding and memorizing the heart structures [4]. Around 40\% of students have misconceptions about heart contraction, and about $56 \%$ of students have misconceptions about the anatomical structure of the heart [5].

The use of a modern learning tool, such as the AR, assists in the development of user-friendly media and multifunctionality. The AR has been used in different health subjects, such as surgery and heart anatomy, to support trainees during their education and training program, since the AR applications assist in information transformation [6]. For example, the usage of the AR helps learners understand and memorize the heart's structures through visual perception, since these concepts are not easy to demonstrate [4]. Research studies on embedded AR software tools reveal their efficacy in students' education. AI is used to explore the use of the AR in medical education, including different forms, student training, patient education, and laboratory simulation. Unique advances in technology and hardware reveal the feasibility of anatomy science education in medical school. Students, alongside their mentors, can easily study the textbook as a 3D object supplemented with images and sounds. The use of the AR applications that support two-dimensional (2D), 3D, audio, and video environments is helpful for learners, since direct observation of the heart and its structures and functions is not possible for students [5].

The AR has also been used to improve physicians' and trainees' visualization in order to enhance patient outcome. The Enhanced Electrophysiology Visualization and Interaction System (ēLVIS) is an AR based system that assists physicians in visualizing and interacting with real data. This system enhances physicians' understanding of a patient's specific cardiac case, which, as stated above, helps in improving the patient's outcome [7]. Similarly, developing an AR mobile application that presents some heart disease cases will improve trainees' understanding and healthcare outcomes [4].

\section{Heart failure cases: Mitral Incompetence and Mitral Stenosis}

Heart failure-also known as congestive heart failure - essentially means that the heart muscle does not pump blood as well as a normal heart. Narrowed arteries in the heart or high blood pressure can cause the heart to be too weak or stiff to fill and pump efficiently [8].

Mitral incompetence occurs when the flaps of the heart's mitral valve bulge like an umbrella into the heart's left upper chamber, the left atrium, as the heart contracts. Mitral valve prolapse sometimes results in blood leaking back into the left atrium. This condition is called mitral incompetence or mitral valve regurgitation. For most people, mitral valve prolapse is not a life-threatening condition and doesn't require medication or modifications to their lifestyle. However, some people need treatment for mitral valve prolapse. Figure 1. shows a comparison between the normal heart and a heart with mitral incompetence [9].

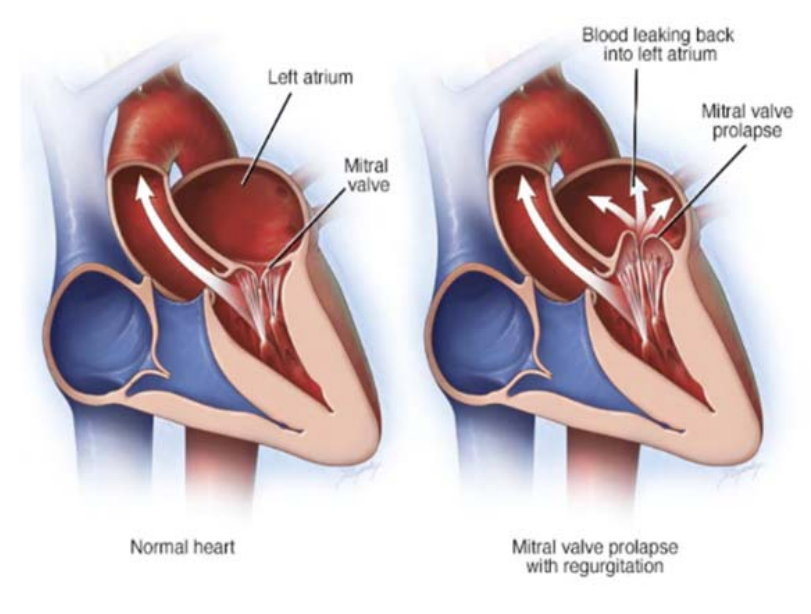

Figure 1. Normal heart and mitral incompetent heart [8]

Mitral stenosis, also called mitral valve stenosis, is a heart failure condition in which a narrowing of the heart's mitral valve occurs. This abnormal valve does not open suitably, preventing the blood from flowing into the main pumping chamber of the heart (left ventricle). Mitral stenosis can make the patient tired and short of breath in addition to other health issues. The main cause of mitral stenosis is an infection called rheumatic fever, which is relevant to strep infections. Mitral stenosis can injure the mitral valve. Left untreated, mitral valve stenosis can lead to serious heart complications. Figure 2. shows a comparison between a normal heart and a heart with mitral stenosis [10]. 


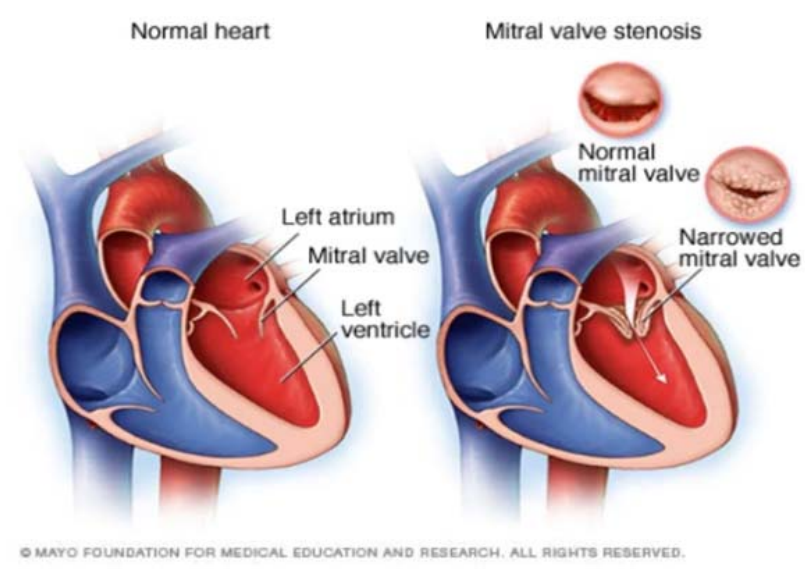

Figure 2. Normal heart and heart with Mitral Stenosis [8]

\section{Related Works}

This section investigates some of the mobile applications that have some functionalities and features similar to CardioSim. Nowadays, smartphones are accessible to everyone and have become a very important element in people's lives [11]. A number of mobile applications have been created to help medical students during their education. However, few of them use the AR and virtual reality (VR) technologies to facilitate learning. The following are some popular applications available on Google Play and the App Store:

1. AR Anatomy 4D+: The main features of this app are AR technology, providing animated images of the main internal organs, and full translation into many languages [12].

2. My Organ Anatomy: This application displays the anatomy of body organs with 3D graphics. It gives the ability to draw on a white screen and share screenshots, and it provides audio pronunciation for all anatomical terms [13].

3. My Heart Anatomy: This application provides audio pronunciation for all heart anatomy terms and gives the users the ability to select any external part of the heart to view the name of the part or read related information about it [14].

Table 1. summarizes the comparison analysis between the CardioSim app and the above applications. For example, it is clear that AR Anatomy 4D + doesn't support the simulation of organ diseases, while the My Organ Anatomy and My Heart Anatomy apps don't provide AR capabilities. Although they are all used for educational purposes, only CardioSim introduces the function of self-assessment with the AR as a result of the semi-structured interviews at the Clinical Skills and Simulation Centre.
Table 1. Comparison analysis between CardioSim App and other applications

\begin{tabular}{|c|c|c|c|c|}
\hline Services & 1 & 2 & 3 & CardioSim \\
\hline AR usage & Yes & No & No & Yes \\
\hline $\begin{array}{l}\text { Coverage of main body } \\
\text { organs }\end{array}$ & Yes & Yes & No & No \\
\hline Anatomy of the organ & Yes & No & Yes & Yes \\
\hline Explaining \& simulating & No & No & No & Yes \\
\hline $\begin{array}{l}\text { Providing organ } \\
\text { information }\end{array}$ & No & No & Yes & Yes \\
\hline $\begin{array}{l}\text { Audio pronunciation of } \\
\text { organ parts }\end{array}$ & No & Yes & Yes & No \\
\hline Self-assessment & No & No & No & Yes \\
\hline
\end{tabular}

\section{Description of the CardioSim App and Clinical Skills Simulation Centre}

CardioSim is an android mobile application that applies interactive AR technology to the medical educational field. It uses AR technology in medical simulation to enhance the learners' education. Students can use it to maintain training on real cases during limited laboratory session time. In order to leverage more productive learning, the use of the AR with $3 \mathrm{D}$ objects that simulate different cases is required. This app focuses on heart anatomy with the support of case descriptions of one type of heart disease - heart failure. CardioSim covers two cases of heart failure, which are mitral incompetence and mitral stenosis. It displays educational videos to show the symptoms and causes of each case. The CardioSim app will be applied and used with one of the manikins at the Clinical Skills Centre and Simulation Centre.

The Clinical Skills Centre and Simulation Centre, King Abdulaziz University, Saudi Arabia, is a stateof-the-art training centre that is a resource for technoscientific training for medical students, nurses, physicians, and other healthcare specialists. At the Centre, participants evaluate and diagnose patients through teaching models that incorporate the use of actor-patients and computerized manikins that simulate disease. The Centre provides opportunities for hands-on training in a safe environment [15]. The Centre has three kinds of manikins: low, middle, and high fidelity. These manikins are categorized into one of the three types according to the degree of realism with which they simulate reality. We conducted several semi-structured interviews with the staff at the centre to identify the main requirements and functions of the app. They emphasized the need for a content on heart failure, as it is a very important learning block in students' training. The learning outcomes that the cardiologists asked to be covered are: causes, symptoms, heartbeat sounds, heart illustrations, and blood flow. These learning objective outcomes are covered for each case of the heart diseases in the CardioSim app. Additionally, the interviewees suggested the addition of the self-assessment component in the app. 


\section{Research Method}

Software development methodology refers to the method of planning, designing, testing, and then deploying a project [16]. The most common methodology used to develop any software is agile, meaning it has the capability to produce and respond to change. It is a way of dealing with, and eventually succeeding in, an uncertain and turbulent environment [17]. In developing CardioSim, we focused on the first two stages in ADR, which are problem formalization and building and evaluating the IT artifact. The authors applied the scrum approach in developing the CardioSim app, which is one of the agile frameworks that is mainly used to manage the product by involving users during development [18].

The CardioSim app has two epics. The stories within the first epic are designing the pages of the application and the functionalities with the selfassessment quizzes for all cases. The second epic contains stories related to the functionalities of using the AR for the heart cases and anatomy. It also contains the functionalities of special self-assessment quizzes for each case.

\section{CardioSim App design}

Through the project lifecycle, we analysed the application using different diagrams, such as use case, workflow, and sequence diagrams. The CardioSim app has two main processes that are represented using a system workflow diagram as shown in Figure 3., while Figure 4. shows the business process model diagram of heart cases. The following are the details of the app's parts description:

1. Heart Cases: Heart cases are displayed whenever a user chooses the first option, which is heart cases. This case has three sub-cases.

1.1 Normal heart: displayed when a user chooses heart cases, then chooses the normal heart. It will display the normal heart and its anatomy.

1.2 Mitral incompetence case: displayed when a user chooses heart cases, then chooses the mitral incompetence case.

1.3 Mitral stenosis case: displayed when a user chooses heart cases, then chooses the mitral stenosis case.
2. Self-Assessment: A self-assessment quiz is started whenever a user chooses the second option, which is self-assessment.

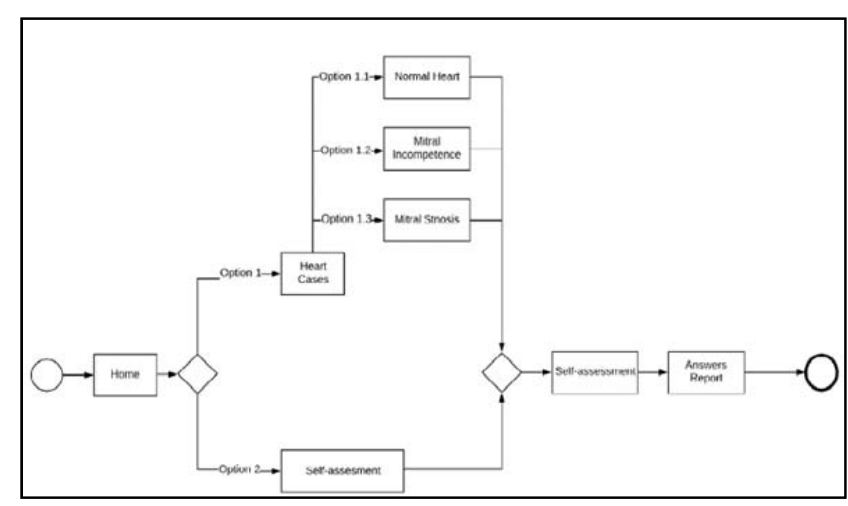

Figure 3. System workflow diagram

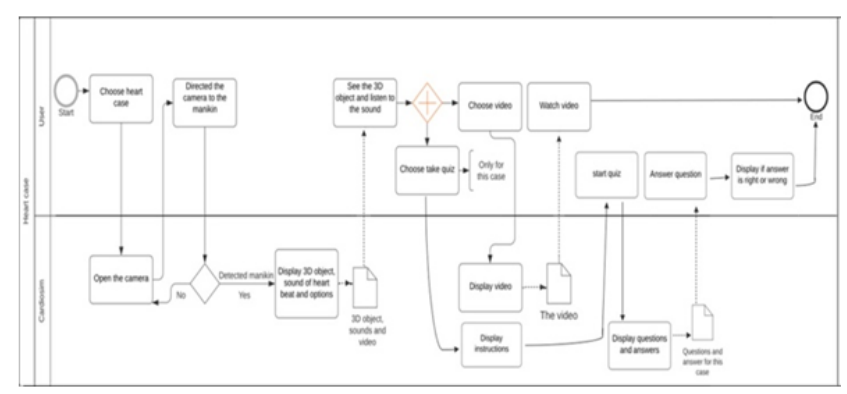

Figure 4. Business process model - heart cases process

\section{Implementation}

We used several software tools to develop the CardioSim app. First, we used Vuforia Engine, which is a platform that helps developers of AR applications add the functionality of advanced computer vision [19]. This allows the app to recognize images and interact with the real world. Second, we used Unity, which is a platform used to develop augmented reality, virtual reality, and mixed reality apps [20]. Third, we used Visual Studio, which is a program used to write the code of the app; we used $\mathrm{CH}$ as a programming language. Fourth, we used Android Studio, which is a program used to extract the app from Unity as an app and upload it to the mobile that runs on the android operating system. The following hardware are needed for this application: (1) a web cam, which is a device used to facilitate app development and testing; (2) a smart mobile phone used to open the camera and direct it to the manikin to display the heart anatomy; (3) a manikin that is available at and provided by the Clinical Skills Centre and Simulation Centre, as shown in Figure 5. 


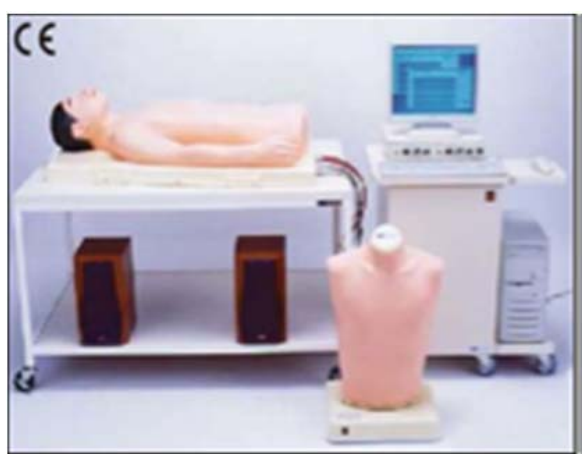

Figure 5. Manikin used with CardioSim at the simulation centre

\section{Testing and Evaluation}

To test and evaluate the app, we applied three techniques: usability testing, code testing, and SUS. We applied these techniques to improve the usability and code quality of the CardioSim app.

\section{Usability Testing}

We applied three of Nielsen's heuristics rules of user interface design during the development of CardioSim. The first rule is to strive for consistency, which is utilized in all CardioSim pages; all pages have the same buttons in the same positions, and the same colour pallet is used [21]. In heart cases and self-assessment, the user will follow the exact same sequence of actions to reach any heart cases or type of self-assessment. The second rule is to enable frequent users to use shortcuts [21]. This rule is applied by enabling multiple ways to reach the help page. The user can reach the homepage from the menu button or by clicking on the "CardioSim" graphic that appears at the top of all pages. The third rule we applied in designing the app interface is to support internal locus of control [21]. In CardioSim, we enable the users to control the sounds. Hence, the user can silence the app at any time. Applying these rules in designing the CardioSim app, assisted in increasing the usability of the app to match the users' requirements.

\section{Code Testing}

The code is tested by two measurements: unit testing and integration testing. Scrum methodology was used to build this application; therefore, unit testing was applied after the completion of each individual story. For example, we have tested CardioSim to verify that a user can: navigate from the homepage to the self-assessment page; navigate from the self-assessment page to the homepage; choose answer A, B, or C; display the answer report of the self-assessment; display the normal heart, mitral incompetence, and mitral stenosis 3D objects; and turn the heartbeats on and off. Lastly, we verified that the user can start the quiz and start and stop all video related to the heart cases. All the results were positive, and the user can complete all scenarios successfully.

Regular integration testing is important in order to assure that the system is error-free. This is performed by checking the interfaces for each use-case with the intent of exposing errors [22]. Conducting both measurements of code testing will ensure that CardioSim is implemented correctly.

The integration testing shows the flow between screens correctly. Figure 6. (a) shows the main page; when the user selects the menu icon, it drops down the menu as shown in Figure 6. (b). The user can select the help option from the menu or from the help icon.

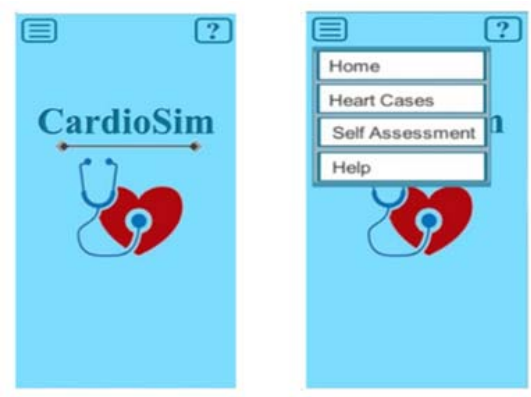

Figure 6. CardioSim app main pages

For the self-assessment, the questions page will display questions in the central area, as shown in Figures 7. (a) and 7. (b). The user can display the questions that have been answered correctly and incorrectly, in addition to the user's score, as shown in Figure 7. (c).
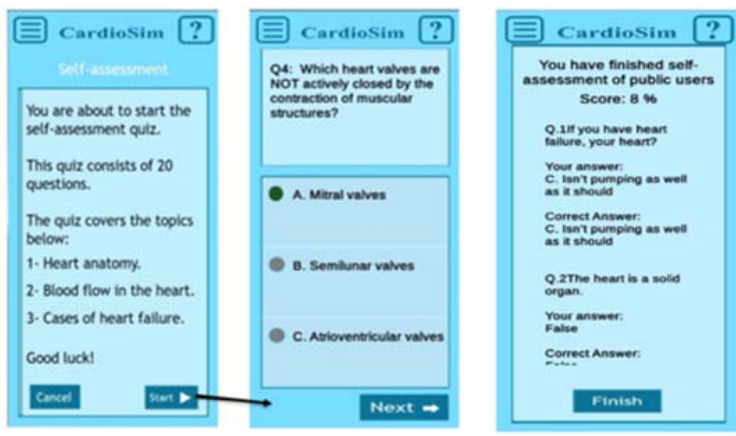

Figure 7. Self-assessment page in CardioSim

The heart cases are similar in design but different in content. There are three options for heart casesnormal heart, mitral incompetence, and mitral stenosis. When the user selects one of them and directs the camera to the manikin, the app will display the 3D heart object that has been designed especially for that case on the manikin, and-at the same time - the sound of the heartbeat of the chosen case will play. In the page of heart cases, there is a 
small window that contains information about the chosen case, and the user can display more information regarding the heart by clicking on the "show more" option, as shown in Figure 8. (a), Figure 8. (b), and Figure 8. (c) for the normal heart, Figure 9. (a), Figure 9. (b), and Figure 9. (c) for mitral incompetence, and Figure 10. (a), Figure 10. (b), and Figure 10. (c) for mitral stenosis.

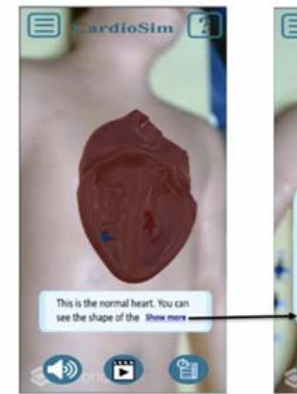

(a)

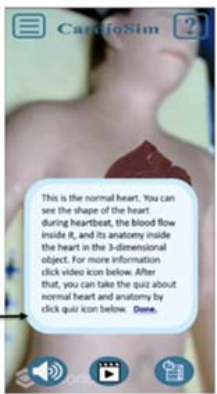

(b)

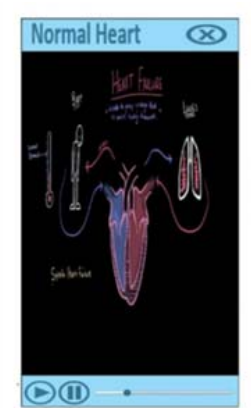

(c)
Figure 8. Normal Heart Case

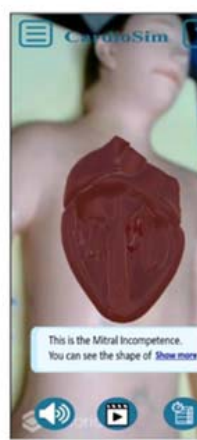

(a)

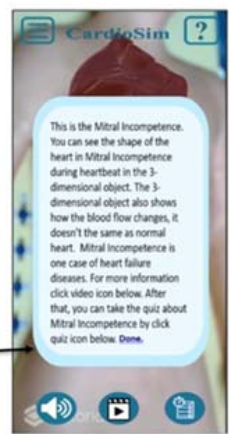

(b)

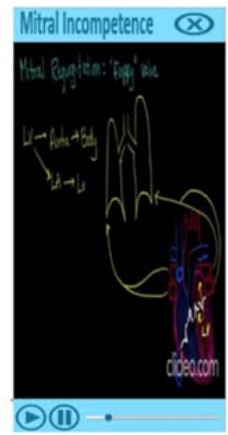

(c)
Figure 9. Mitral Incompetence Case

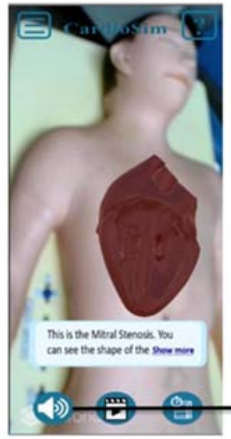

(a)

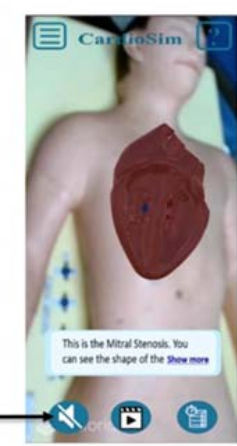

(b)

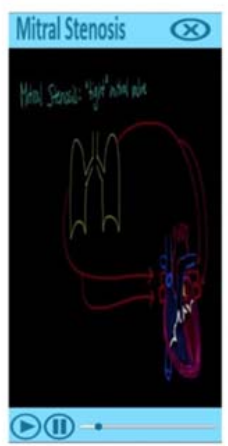

(c)
Figure 10. Mitral Stenosis Case

Also, there are three options at the bottom of each page. The first option is to turn the heartbeat sound on and off. The second option is to start watching the informatic video that contains more information about the chosen case, such as describing symptoms and causes, as shown in Figures 8. (c), Figure 9. (c), and Figure 10. (c). The third option is to start a selfassessment quiz that covers what the user has learned during the session.
This type of testing allows us to test and navigate the transactions between different units of the CardioSim app. We tested each epic; all the results were positive.

\section{System Usability Scale (SUS)}

SUS is one of the most commonly used instruments for measuring the perceived usability of a system or product. John Brooke initially created it while working on digital equipment. It consists of 10 item questionnaires to which users rate their agreement level. Half the questionnaires are worded positively, and the other half are worded negatively. For each agreement, a five-point scale is used [23]. We used SUS to test the usability of the CardioSim app. We recruited 10 participants using a convenience and snowball sampling technique; the participants were medical students from the centre. After collecting the data, we analysed it, and the score of the SUS was 76.75, which is acceptable and ranks as good based on the SUS grading scores [24].

\section{Release of liability}

A release of liability agreement is a legal document between two parties in which the releasor or person agrees not to sue. In our case, to avoid any misuse of the CardioSim app, we wrote this release of liability agreement that appears at the beginning of the CardioSim app, as shown in Figure 11.

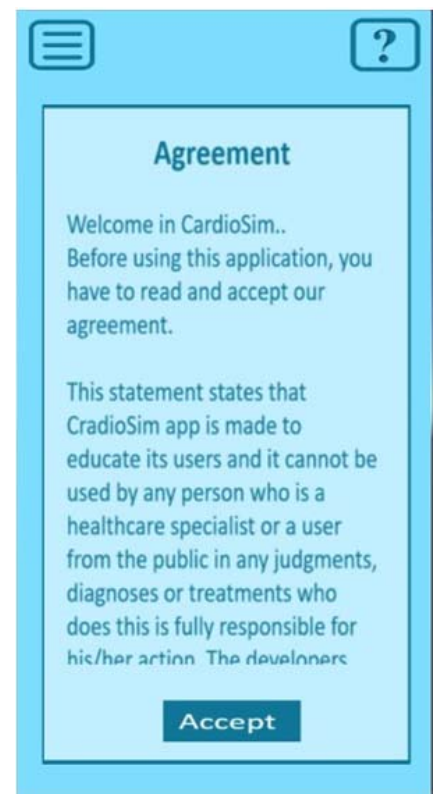

Figure 11. Release of Liability Statement Page

\section{Discussion, Limitations, Future Works, and Conclusions}

The CardioSim app will enable better understanding and a more attractive and effective method of learning through the use of one of the most 
promising modern technologies in education-AR. CardioSim simulates the healthy heart and two heart failure cases and their associated heartbeats as requested by the Clinical Skills and Simulation Centre's staff at King Abdulaziz University Hospital. Assessment is also implemented based on one of the student learning outcomes. Using this app, the user can learn more about these cases interactively. ADR reflects the promise of the IT artifact, the CardioSim app, since the development and the evaluating phases were performed in the organizational context, although more evaluation is required. ADR helped the development team understand the research's focus and scope. Through the CardioSim app lifecycle, we addressed the need to understand the heart and some of its diseases using AR techniques and also evaluated the app using SUS measurement. These two phases-problem formulation and building and evaluating an IT artifact in an organizational context - reflected just two ADR stages [25].

This research project is still an ongoing ADR, as more evaluation is needed. The remaining two stages of ADR - reflection and learning and formalization of learning - must be finalized. One of the research limitations is the evaluation - the researchers only evaluated the usability of the app. Therefore, in future work, the authors will evaluate the app's effectiveness using both quantitative and qualitative methods involving both healthcare professionals and trainees from the Centre. The authors will use a validated instrument to evaluate the app if such an instrument exists; if not, they will develop and validate an instrument. Furthermore, the authors will work and finalize this ADR focusing on all the stages as mentioned in Sein et al.'s Action Design Research [25].

The optimization of AR in education and training has been clearly discussed in early research. The widespread usage of this technology among several disciplines, such as business, industry, health, and education, has been beneficial. This modern technology assists in solving several issues, such as lacking resources, by providing, for example, information in computer-generated $3 \mathrm{D}$ imagery at the right time and place. This technology, as shown in this research project, could be usefully applied for trainees in any health training institution.

\section{References}

[1]. Yuen, S. C. Y., Yaoyuneyong, G., \& Johnson, E. (2011). Augmented reality: An overview and five directions for AR in education. Journal of Educational Technology Development and Exchange (JETDE), 4(1), 11.

[2]. Viggers, S., Østergaard, D., \& Dieckmann, P. (2020). How to include medical students in your healthcare simulation centre workforce. Advances in Simulation, 5(1), 1-6.

[3]. World Health Organization. (n.d). Cardiovascular Diseases. Retrieved from: https:/www.who.int/health-topics/cardiovasculardiseases\#tab=tab 1 [accessed: 10 September 2020].

[4]. Nuanmeesri, S., Kadmateekarun, P., \& Poomhiran, L. (2019). Augmented Reality to Teach Human Heart Anatomy and Blood Flow. Turkish Online Journal of Educational Technology-TOJET, 18(1), 15-24.

[5]. Celik, C., Guven, G., \& Cakir, N. K. (2020). Integration of mobile augmented reality (MAR) applications into biology laboratory: Anatomic structure of the heart. Research in Learning Technology, 28.

[6]. Barsom, E. Z., Graafland, M., \& Schijven, M. P. (2016). Systematic review on the effectiveness of augmented reality applications in medical training. Surgical endoscopy, 30(10), 4174-4183.

[7]. Prakosa, A., Southworth, M. K., Silva, J. N. A., Silva, J. R., \& Trayanova, N. A. (2021). Impact of augmented-reality improvement in ablation catheter navigation as assessed by virtual-heart simulations of ventricular tachycardia ablation. Computers in Biology and Medicine, 133, 104366.

[8]. Mayo Clinic. (n.d). Heart failure - Symptoms and causes. Retrieved from:

https://www.mayoclinic.org/diseasesconditions/heart-failure/symptoms-causes/syc$\underline{20373142}$ [accessed: 17 September 2020].

[9]. Mayo Clinic. (n.d). Mitral valve regurgitation. Retrieved from:

https://www.mayoclinic.org/diseasesconditions/mitral-valve-regurgitation/symptomscauses/syc-20350178 [accessed: 17 September 2020].

[10]. Mayo Clinic. (n.d). Mitral valve stenosis. Retrieved from: https://www.mayoclinic.org/diseasesconditions/mitral-valve-stenosis/symptomscauses/syc-20353159 [accessed: 12 October 2020].

[11]. Garner, S. L., George, C. E., Young, P., Hitchcock, J., Koch, H., Green, G., ... \& Norman, G. (2020). Effectiveness of an mHealth application to improve hypertension health literacy in India. International Nursing Review, 67(4), 476-483.

[12]. Begic, M., Cirimotic, M., Farkas, I., Skoric, I., Car, Z., Rasan, I., \& Zilak, M. (2020). Software prototype based on augmented reality for mastering vocabulary. In 2020 43rd International Convention on Information, Communication and Electronic Technology (MIPRO) (pp. 446-451). IEEE. 
[13]. Pellerin, M., Vergote, D., Cervantes, V. F., Ogandaga, M., DeJesus, R., \& Stroulia, E. (2020, November). Designing an online interactive and bilingual 3D learning environment for human anatomy education. In Innovate Learning Summit 2020 (pp. 581-590). Association for the Advancement of Computing in Education (AACE).

[14]. Krishnasamy, S., Mokhtar, R. A. R., Singh, R., Sivallingam, S., Aziz, Y. F. A., \& Mathaneswaran, V. (2021). 3D Rapid Prototyping Heart Model Validation for Teaching and Training-A Pilot Project in a Teaching Institution. Brazilian journal of cardiovascular surgery.

[15]. King Abdulaziz University. (n.d). About Clinical skills and simulation center. Retrieved from: https://csc.kau.edu.sa/Pages-About.aspx [accessed: 15 October 2020].

[16]. Saeed, S., Jhanjhi, N. Z., Naqvi, M., \& Humayun, M. (2019). Analysis of Software Development Methodologies. International Journal of Computing and Digital Systems, 8(5), 446-460.

[17]. Venkatesh, V., Thong, J. Y., Chan, F. K., Hoehle, H., \& Spohrer, K. (2020). How agile software development methods reduce work exhaustion: Insights on role perceptions and organizational skills. Information Systems Journal, 30(4), 733-761.

[18]. Maier, P., Ma, Z., \& Bloem, R. (2017, August). Towards a secure scrum process for agile web application development. In Proceedings of the 12th International Conference on Availability, Reliability and Security (pp. 1-8).

[19]. Linowes, J., \& Babilinski, K. (2017). Augmented reality for developers: Build practical augmented reality applications with unity, ARCore, ARKit, and Vuforia. Packt Publishing Ltd.

[20]. Unity. Unity for VR and AR. (n.d). Retrieved Jul 21, 2020, Retrieved from: https://unity3d.com/unity/features/multiplatform/vrar $\quad$ [accessed: 25 October 2020].

[21]. Ranzani, F., \& Parlangeli, O. (2021). Digital Technology and Usability and Ergonomics of Medical Devices. In Textbook of Patient Safety and Clinical Risk Management (pp. 455-464). Springer, Cham.

[22]. O'Regan, G. (2019). Fundamentals of software quality. In Concise Guide to Software Testing (pp. 131). Springer, Cham.

[23]. Albert, W., \& Tullis, T. (2013). Measuring the user experience: collecting, analyzing, and presenting usability metrics. Newnes.

[24]. Kaya, A., Ozturk, R., \& Gumussoy, C. A. (2019). Usability measurement of mobile applications with system usability scale (SUS). In Industrial engineering in the big data era(pp. 389-400). Springer, Cham.

[25]. Sein, M. K., Henfridsson, O., Purao, S., Rossi, M., \& Lindgren, R. (2011). Action design research. MIS quarterly, 37-56.

\section{Appendix}

Supplementary Materials: In CardioSim, we used informatics videos for each heart case-normal, mitral stenosis, and mitral incompetence - and two photos for mitral stenosis and mitral incompetence, which contain more information about the cases. All the videos and photos were approved by the cardiologist Dr. Wael Wahed. The videos are available online at:

https://www.khanacademy.org/science/health-andmedicine/circulatory-system-diseases/heart-valvediseases/v/mitral-valve-regurgitation-and-mitral-valveprolapse

https://www.khanacademy.org/science/health-andmedicine/circulatory-system-diseases/heart-valvediseases $/ \mathrm{v} / \mathrm{mitral}$-stenosis

and

https://www.khanacademy.org/science/health-andmedicine/circulatory-system/circulatory-systemintroduction/v/flow-through-the-heart

[accessed: 20 November 2020]. 